\title{
Realization of Radar Warning Receiver Simulation System
}

\author{
Liu Limin, Cheng Cheng and Han Zhuangzhi \\ (Department of Electronic and Optical Engineering, Mechanical Engineering \\ College, 050003, Shijiazhuang Hebei) \\ 369519528@qq.com
}

\begin{abstract}
According to the structure of the mainstream modern airborne radar warning receiver, a modeling method of radar warning receiver simulation system is presented in this paper, and it realized under the condition of SystemVue. The system consist of radar signal interception, parameter estimation and signal sorting, pulse description words can be obtained at last. The application of parameter estimation method, based on cyclic correlation, and the sorting method, based on detection of single source point, improve the robustness of whole simulation system. Finally, experiment results verify the feasibility of the scheme.
\end{abstract}

Keywords: Radar Warning Receiver; Direction of Arrival (DOA); Time-Frequency Single Source Point; Cyclic correlation; SystemVue

\section{Introduction}

Under the condition of modern war, combat aircraft is faced with an unpredictable environment filled with known and unknown signals that may include target returns, clutter, jamming, interference, and electromagnetic noise, it cannot live unless grasping the whole tactical situation and discovering threats in time. Radar warning receiver (RWR) is one of the radar countermeasure equipments, it is used for the interception, analysis and recognition of radar signals in the air. Through passive-measuring and analyzing the radar waves to provide orientation, threat types and operative mode to pilot. So radar warning receiver has became an indispensable electronic warfare equipment, and it is necessary to research on radar warning receiver. The design scheme of radar warning receiver modeling system is presented in this paper, the system simulates the structure characteristics and data flow of RWR, every task of real RWR can be realized in the system. The advantage of simulation system is stronger extensibility, more flexible operation and lower cost. The validity or superiority of algorithm can be verified in the modeling system, and it also can be used as a subsystem applied to larger, more complex modeling system.

In this paper, we use SystemVue to establish the RWR simulation system. SystemVue is a combination of commercial, off-the-shelf software and instrumentation from Agilent. It is easier to create realistic signal scenarios such as multi-radar signals in a complex electromagnetic environment. And it can combine with hardware, this combination provides a platform that can be used for both component testing and scenario simulation for system test, such as the addition of a signal analyzer or wideband oscilloscope running the vector signal analysis (VSA) software provides measurement and analysis capabilities that are useful in the development of transmitters, receivers, amplifiers, and other subsystems. There are two innovative methods, the joint estimation method of DOA and signal numbers based on cyclic correlation and the signal sorting method based on detection of time-frequency single source point, apply to the system to improve the robustness and estimate accuracy. 


\section{Structure}

Now, the radar warning receiver is a high performance system with respect to detection range, selectivity and processing power. Such as BOW, the mainstream of radar warning receiver system, has been used in "Tornado" and "Gripen" aircraft. Excellent performance in combination with a modular design and very good growth potential make the BOW one of the most powerful RWR systems presently available. There are two parts in BOW, front intercept and signal sorting. The front intercept part includes three modules: monopulse antennas, wideband receiver and narrowband receiver, has the effect of intercepting airspace signal within the scope of search frequency domain, and the intercepted signal is sent to the signal sorting part. The signal sorting part includes two modules: pulse processor and radar warning computer. In this part, target signals can be sorted out from the mixed signals and the alarm signals are produced out.

According to the structure of the BOW system, a design scheme of RWR modeling system is proposed in this paper. It includes three parts: front signal intercept, parameter estimation and signal sorting. In this modeling system, the input is mixed signals, and the output is pulse description words (PDW): direction of arrival, time of arrival, pulse width, carrier frequency and amplitude.

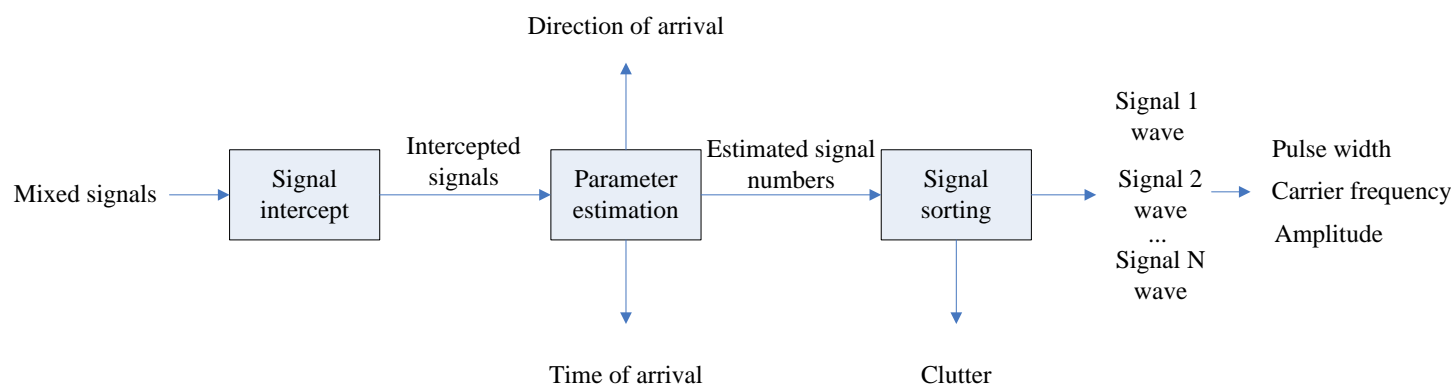

Figure 1. Design Scheme of Modeling RWR System

As we can see in Figure 1, three of the PDW are obtained from signal waves. Traditional estimation of PDW is to contrast the pulse description words in radar pulse library, so that the sorting speed is slower. In this signal sorting module, the sorting method, in combination with blind signal separation (BSS), can estimate time-domain wave of each radar source signal accurately, and the PDW is acquired from signal waves. The new method can achieve high speed signal sorting.

Table 1. Input and Output of Each Module

\begin{tabular}{|c|c|c|c|}
\hline \multicolumn{2}{|c|}{ Module } & Input & Output \\
\hline \multicolumn{2}{|c|}{ Signal intercept } & Mixed signals & Intercepted signals \\
\hline \multirow{3}{*}{ Parameter estimation } & DOA estimation & \multirow{3}{*}{ Intercepted signals } & Direction of arrival \\
\hline & TOA estimation & & Time of arrival \\
\hline & $\begin{array}{c}\text { Signal numbers } \\
\text { estimation }\end{array}$ & & Source number \\
\hline \multirow{4}{*}{\multicolumn{2}{|c|}{ Signal sorting }} & \multirow{4}{*}{$\begin{array}{l}\text { Intercepted signals \& } \\
\text { Source number }\end{array}$} & Signal waves \\
\hline & & & Pulse width \\
\hline & & & Carrier frequency \\
\hline & & & Amplitude \\
\hline
\end{tabular}

The data flow shown in Table 1. We can see that our modeling system can work out each PDW from the mixed signals, that is to say the system can accomplish the task of RWR. 


\section{Modules}

Mentioned above are analyzed theoretically the feasibility of the simulation system, in this section, we start to study on how to realize each module.

\subsection{Signal Intercept Module}

Whether the signal can be intercepted is determined by various threshold: frequency-domain intercepting threshold, signal energy threshold and airspace intercepting threshold. If one signal meets the requirements of the above three kinds of threshold, can we identify this signal is intercepted. Threshold decision diagram is shown in Figure 2, and specific process is shown in Figure 3.

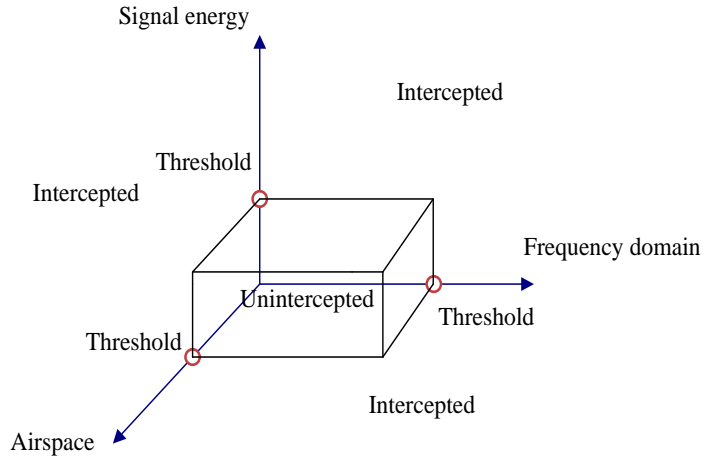

Figure 2. Threshold Decision Diagram

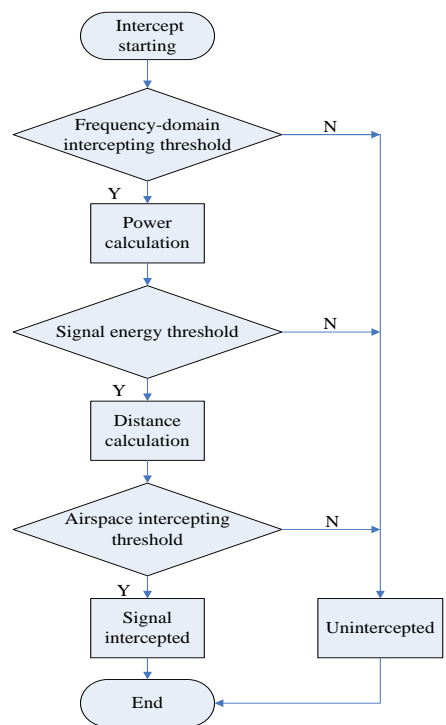

Figure 3. The Flow of Intercepting Decision Process

In Figure 2, three axes represent three domains respectively, three points represent three threshold values. So one signal, in the cuboid, is regarded as unintercepted signals, and out of the cuboid is regarded as the area that signals can be intercepted. Then the intercepted signals are sampled by this module, and the intercepted signals, in the form of data, are passed to the next module.

\subsection{Parameter Estimation Module}

The method of TOA estimation, same as the method in literature [4], based on Correlation and Reversed Accumulation, we do not discuss in this paper. We focus on the estimate method of DOA and source numbers. In this paper, a joint estimate method based on cyclic correlation is proposed to estimate DOA and numbers. Here is the concrete implementation process.

Suppose the number of radar signals is $P$, the number of antenna array is $M$. So the intercepted signal or observed signal can be described as

$$
x(t)=A s(t)+n(t)
$$

Where $x(t)$ is observed signals, $x(t)=\left[x_{1}(t), x_{2}(t), \ldots, x_{M}(t)\right]^{T} ; s(t)$ is radar source signals, $\quad s(t)=\left[s_{1}(t), s_{2}(t), \ldots, s_{P}(t)\right]^{T} ; n(t) \quad$ is antenna noise, $n(t)=\left[n_{1}(t), n_{2}(t), \ldots, n_{M}(t)\right]^{T} ; A$ is mixed matrix, $A=\left[\alpha_{1}, \alpha_{2} \ldots, \alpha_{P}\right]^{T}$.

The mean of $x(t)$ can be expressed as 


$$
M_{x}(t)=E[x(t)]
$$

Obviously, $M_{x}(t)$ is the time function, so we can not use the average time to estimate the mean of $x(t)$. We use sample mean to estimate the mean

$$
M_{x}(t)=E[x(t+n T)]=\lim _{N \rightarrow \infty} \frac{1}{2 N+1} \sum_{n=-N}^{N} x(t+n T)
$$

Where $T$ is the cycle of the signal; $M_{x}(t)$ is periodic function. Taking the Fourier series expansion of (3), we obtain

$$
M_{x}(t)=\sum_{m=-\infty}^{+\infty} M_{x}^{m / T} e^{j 2 \pi m t / T}
$$

The corresponding Fourier coefficient $M_{x}^{m / T}$ is

$$
M_{x}^{m / T}=\frac{1}{T} \int_{-T / 2}^{T / 2} M_{x}(t) e^{-j 2 \pi m t / T} d t=\left\langle x(t) e^{-j 2 \pi m t / T}\right\rangle_{t}
$$

Where \langle\rangle$_{t}$ represents the average of time, so the Fourier coefficient becomes

$$
M_{x}^{\varepsilon}(t)=\left\langle x(t) e^{-j 2 \pi \varepsilon t}\right\rangle_{t}
$$

Where $\varepsilon$ is harmonic component; $M_{x}^{\varepsilon}(t)$ is called cycle mean.

Definition 1. Cyclic-correlation function

The tine-varying correlation function of $x(t)$ is

$$
R_{x}(t, \tau)=E\left\{x(t) x^{*}(t+\tau)\right\}
$$

Based on the previous known, we use the time average to figure out the mean of $x(t) x^{*}(t+\tau)$, so $R_{x}(t, \tau)$ becomes

$$
R_{x}(t, \tau)=\lim _{N \rightarrow \infty} \frac{1}{2 N+1} \sum_{n=-N}^{N} x\left(t+n T_{0}\right) x^{*}\left(t+n T_{0}+\tau\right)
$$

Taking the Fourier series expansion of (8), we obtain

$$
R_{x}^{\alpha}(\tau)=\frac{1}{T_{0}} \int_{-T_{0} / 2}^{+T_{0} / 2} R_{x}(t, \tau) e^{-j 2 \pi \alpha t} d t
$$

So the cyclic-correlation function of observed signals $x(t)$ is

$$
R_{x}^{\alpha}(\tau)=\left\langle x(t) x^{*}(t+\tau) e^{-j 2 \pi \alpha t}\right\rangle_{t}
$$

In this paper, suppose radar signals is linear frequency-modulated (LFM) signals, so the signals can be described as

$$
s(t)=e^{j\left(\omega_{0} t+\omega_{1} t^{2}+\theta_{0}\right)}
$$

Where $\omega_{0}$ is carrier frequency, $\omega_{1}$ is modulation rate, $\theta_{0}$ is initial phase. In order to simplify the derivation, $\theta_{0}=0$ in this paper. Bringing Eq. (1) and (11) into Eq. (10), we obtain

$$
R_{x}^{\alpha}(\tau)=\sum_{m=1}^{M} \sum_{n=m} f_{1}(\tau) \delta\left[2 \pi \alpha+2 \omega_{1} \tau\right]+\sum_{m=1}^{M} \sum_{n \neq m} f_{2}(\tau) \delta\left[2 \pi \alpha+2 \omega_{1}\left(\tau+\tau_{m}-\tau_{n}\right)\right]+f_{n}(\tau) \delta(\alpha)
$$

Where $f(\tau)$ is the function of $\tau$. In Eq. (12), we focus on the value of cyclic correlation frequency $\alpha$, so we do not consider the specific expression of $f(\tau)$. Due to the property of function $\delta(x)$, if and only if $x=0, \delta(0)=1$. So the nonzero values appear in the condition of $\delta(x)=1$, when $\alpha=-\omega_{1}\left(\tau+\tau_{m}-\tau_{n}\right) / \pi$ or $\alpha=-\omega_{1} / \pi$, so 
$\delta(\alpha)=0$, value of the last item is zero, noise is eliminated. So the rest nonzero is generated by signals, we can obtain the signal numbers from the spatial spectrum. The abscissa of spatial spectrum represents cyclic correlation frequency, and the cyclic correlation frequency can be described as

$$
\alpha_{m}=\arg \max \left|R_{x}^{\alpha}(l)\right|
$$

Where $\alpha_{m}$ is the cyclic correlation frequency corresponding to the peak values of spatial spectrum, so the DOA is the angle information of $\alpha_{m}$, we can figure out by Eq. (13).

\subsection{Signal Sorting Module}

Suppose the time-frequency single source points set of signal $s_{k}(t)$ is $\Psi\left(t_{k_{i}}, f_{k_{i}}\right)$. So observed signal of any point in the assemblage can be described as

$$
x(t, f)=a_{k} s_{k}(t, f)+n(t, f)
$$

Definition 2. Time-frequency support point.

If $\|x(t, f)\|_{2}^{2}>0$, point $(t, f)$ is the time-frequency support point of $x(t, f)$. When it considers the antenna noise, the criterion of time-frequency support point becomes to $\|x(t, f)\|_{2}^{2}>\xi$, where $\xi$ is noise gate.

Definition 3. Time-frequency single source point.

In the time-frequency plane, if $s_{i}(t, f) \square s_{k}(t, f) \quad i \neq k$, we consider that at $(t, f)$ point, there only exist $s_{i}(t, f)$, point $(t, f)$ is the time-frequency single source point of $s_{i}(t, f)$.

Ignoring the influence of noise, Eq. (14) is simplified to

$$
x(t, f)=a_{k} s_{k}(t, f)
$$

Calculating each channel and the $m$ channel time-frequency ratio

$$
\omega=\left[\frac{x_{1}(t, f)}{x_{m}(t, f)}, \ldots, 1, \frac{x_{m+1}(t, f)}{x_{m}(t, f)}, \ldots, \frac{x_{M}(t, f)}{x_{m}(t, f)}\right]
$$

Bringing Eq. (15) into Eq. (16), we obtain

$$
\omega=\left[\frac{\alpha_{k 1}}{\alpha_{k m}}, \ldots, 1, \frac{\alpha_{k(m+1)}}{\alpha_{k m}}, \ldots, \frac{\alpha_{k M}}{\alpha_{k m}}\right]=\frac{1}{\alpha_{k m}} \alpha_{k}
$$

Eq. (17) indicates that if point $(t, f)$ is one time-frequency single source point of signal $s_{k}(t)$, the time-frequency ratio is constant. So we can get the estimation of vector via detecting all time-frequency single source points. The estimation of vector is

$$
\hat{\alpha}_{k}=\left[\frac{1}{L_{k}} \sum_{i=1}^{L_{k}} \frac{x_{1}\left(t_{k_{i}}, f_{k_{i}}\right)}{x_{m}\left(t_{k_{i}}, f_{k_{i}}\right)}, \ldots, \frac{1}{L_{k}} \sum_{i=1}^{L_{k}} \frac{x_{M}\left(t_{k_{i}}, f_{k_{i}}\right)}{x_{m}\left(t_{k_{i}}, f_{k_{i}}\right)}\right]
$$

Where $L_{k}$ is the number of single source points. If we consider the array noise, $\omega$ is not a constant, but mixed signal has obvious clustering characteristics, we can statistical detect single source points.

Considering array noise, the matrix of time-frequency ratio becomes a complex matrix. So we take the real part and the imaginary part into the histogram statistics respectively to get the matrix. Firstly, extract the real and imaginary parts of each element in the matrix. Then, divide the real and imaginary parts into $M_{1}$ and $M_{2}$ groups respectively. The column vector corresponding to each group becomes submatrix. At last, remove the submatrix which number of column less than $K_{1}$ and $K_{2}$, and the rest of submatrix represent to $R_{j k}$ and $I_{j k}$. So the 
time-frequency single source points assemblage corresponding to $R_{j k}$ and $I_{j k}$ is come from one radar source signal.

For example, when $m=1$, the corresponding matrix of time-frequency ratio is

$$
\tilde{\omega}=\left[\begin{array}{cccc}
1 & 1 & \ldots & 1 \\
\frac{x_{2}\left(\tilde{t}_{1}, \tilde{f}_{1}\right)}{x_{1}\left(\tilde{t}_{1}, \tilde{f}_{1}\right)} & \frac{x_{2}\left(\tilde{t}_{2}, \tilde{f}_{2}\right)}{x_{1}\left(\tilde{t}_{2}, \tilde{f}_{2}\right)} & \ldots & \frac{x_{2}\left(\tilde{t}_{N_{i}}, \tilde{f}_{N_{i}}\right)}{x_{1}\left(\tilde{t}_{N_{i}}, \tilde{f}_{N_{i}}\right)} \\
\ldots & \ldots & \ldots & \ldots \\
\frac{x_{M}\left(\tilde{t}_{1}, \tilde{f}_{1}\right)}{x_{1}\left(\tilde{t}_{1}, \tilde{f}_{1}\right)} & \frac{x_{M}\left(\tilde{t}_{2}, \tilde{f}_{2}\right)}{x_{1}\left(\tilde{t}_{2}, \tilde{f}_{2}\right)} & \ldots & \frac{x_{M}\left(\tilde{t}_{N_{i}}, \tilde{f}_{N_{i}}\right)}{x_{1}\left(\tilde{t}_{N_{i}}, \tilde{f}_{N_{i}}\right)}
\end{array}\right]
$$

The Eq. (18) becomes

$$
\hat{e}_{k}=\left[\frac{1}{L_{k}^{\prime}} \sum_{i=1}^{L_{k}^{\prime}} \tilde{\omega}_{i}(1, t), \ldots, \frac{1}{L_{k}^{\prime}} \sum_{i=1}^{L_{k}^{\prime}} \tilde{\omega}_{i}(M, t)\right]
$$

Then work out the autocorrelation matrix of the mixed signal, the autocorrelation matrix is

$$
\tilde{R}_{i}=E\left\{\tilde{x} \tilde{x}^{H}\right\}
$$

Where the superscript " $\mathrm{H}$ " denotes the Hermitian transpose operation. And the Singular Value Decomposition of $\tilde{R}_{i}$ is computed through

$$
\tilde{R}_{i}=U S U^{H}
$$

Where $U$ is a unitary matrix correspondings to the singular value matrix $S$, and $U=\left[u_{1}, u_{2}, \ldots u_{M}\right]$.

From the above, only one signal exists at the time-frequency single source point, and combining with the characteristics of singular value decomposition, if there is one signal, the feature vector correspondings to the maximal eigenvalue in the singular value matrix $S$ is the estimation of mixed vector. So the estimation of mixed vector is

$$
\hat{e}_{k}=u_{\text {Smax }}
$$

Where $u_{\mathrm{Smax}}$ is a vector corresponding to the maximal eigenvalue in unitary matrix $U$.

All above is just the situation of $m=1$, so we should change the value of $m$ from 1 to $M$, repeat the above process, then all the mixed vector can be worked out. The estimation method of vector need go though all the value of $m$, the result is that the vectors of mixed matrix $A$ are estimated too many times. In order to estimate $A$, we must clustering all the estimated vectors. Because the number of signals has been estimated out in parameter estimation module, so we use k-means clustering algorithm to cluster estimated vectors, the matrix $A$ is composed of clustered vectors.

\section{Simulation}

The simulations are performed using MATLAB R2011b and SystemVue 2013. 08 , running on an Inter (R) Core (TM) i3-4150, 3.50 GHz processor with $4 \mathrm{~GB}$ of memory, under Windows 7 OS.

\subsection{Mixed Signals Generation}

Radar Model Library in SystemVue is an advanced simulation block set of over 35 highly-parameterized primitive blocks and higher-level reference designs. It can be used for modeling different types of radar systems, creating radar signal processing algorithms, evaluating system's performance and providing proof-of-concept designs. The block set and its example workspaces serve as algorithmic and architectural reference designs to 
verify radar performance under different conditions. These include target and radar cross section (RCS) scenarios, clutter conditions, jamming (intentional) and environmental interference, and the effect of various receiver algorithms. It is ideal for radar designers who need to generate precise signals for algorithm and hardware verification, or study the performance of their radar systems under various conditions. So we use the radar library to generate radar signals.

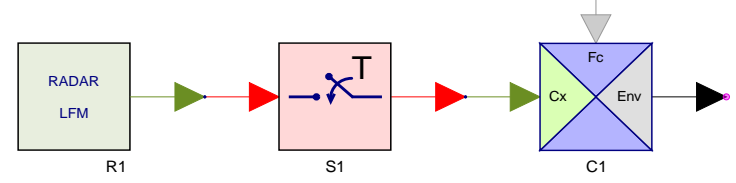

Figure 4. LFM Signal Generation

As we can see in Figure 4, we can generate LFM signals by "RADAR LFM" model, and there are six parameters can be set up, includes Pulsewidth, PRI, PRI_combination, Bandwidth, FM_offset and BB_samplingRate. And the generated LFM signals need to up conversion to form the RF signals.

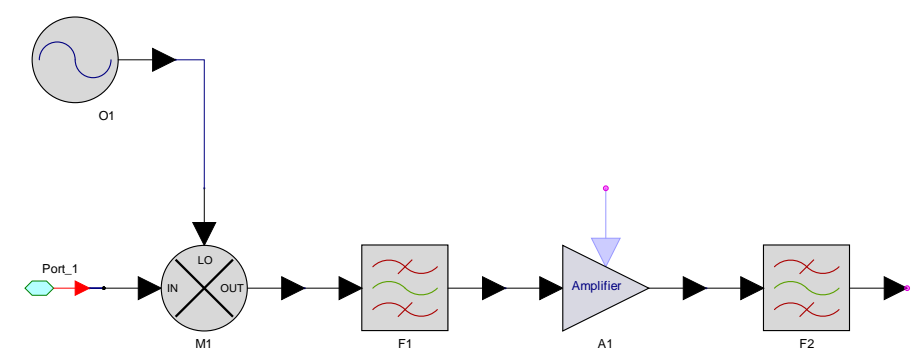

\section{Figure 5. RF Section of Radar Transmitter using the RF Block Library in SystemVue}

Where "Port_1" represents LFM signals, "O1" is an oscillator, "M1" is a mixer, "F1", "F2" are bandpass bessel filters, "A1" is an amplifier. The output is the simulation of radar transmitting signals. In the system, we use different magnification reflect the mixed matrix.

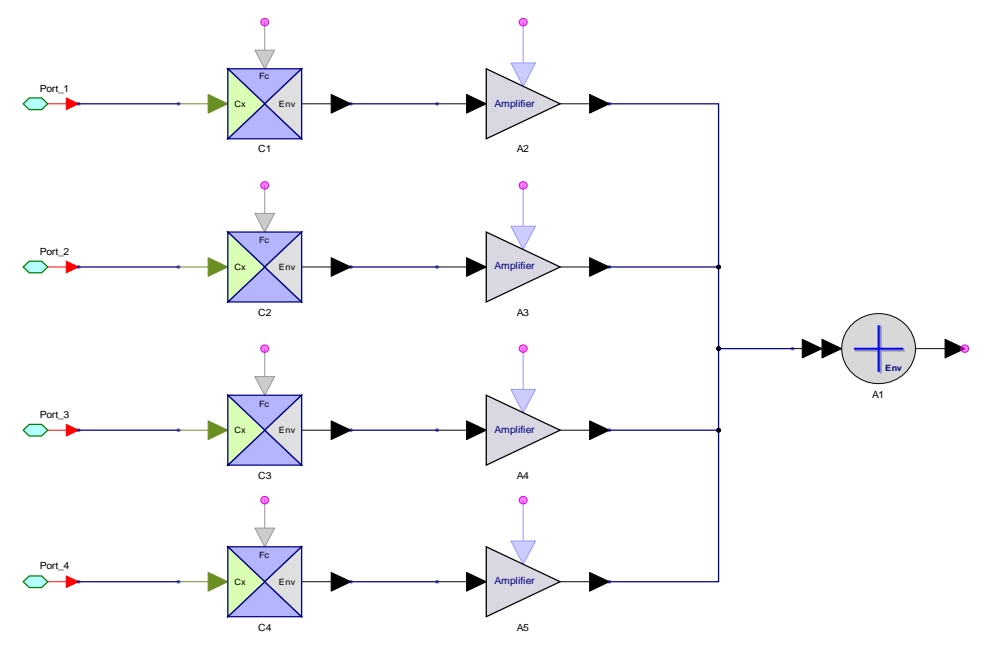

Figure 6. Implementation of Mixed Signals 
Where "Port" represent different RF signals. Suppose magnification is $A_{m}$, so the No. $k$ observation channel corresponding amplification matrix is

$$
A_{k}=\left[A_{k 1}, A_{k 2}, \ldots, A_{k P}\right]
$$

And the mixed matrix can be described as

So the mixed signals is

$$
A=\left[\begin{array}{cccc}
A_{11} & A_{12} & \ldots & A_{1 P} \\
A_{21} & A_{22} & \ldots & A_{2 P} \\
\ldots & \ldots & \ldots & \ldots \\
A_{M 1} & A_{M 2} & \ldots & A_{M P}
\end{array}\right]
$$

$$
\text { Mixed_Signals }=\left[\begin{array}{cccc}
L F M_{1} \cdot A_{11} & L F M_{2} \cdot A_{12} & \ldots & L F M_{P} \cdot A_{1 P} \\
L F M_{1} \cdot A_{21} & L F M_{2} \cdot A_{22} & \ldots & L F M_{P} \cdot A_{2 P} \\
\ldots & \ldots & \ldots & \ldots \\
L F M_{1} \cdot A_{M 1} & L F M_{2} \cdot A_{M 2} & \ldots & L F M_{P} \cdot A_{M P}
\end{array}\right]
$$

Where each row vector represents one observation channel.

\subsection{Experiment}

Suppose there are 4 LFM signals, 3 antenna arrays. The parameters of LFM signals are

Table 2. LFM Signal Parameters

(a) Radar_signal_1

\begin{tabular}{ccc}
\hline Name & Value & Units \\
& & \\
\hline Smple_frequency & 5 & $\mathrm{KHz}$ \\
Sampling_points & 1000 & $($ ) \\
Starting_frequency & 100 & $\mathrm{~Hz}$ \\
Modulation_rate & 20 & $($ ) \\
DOA & -42 & $\circ$ \\
\hline (b) Radar_signal_2 & \\
\hline Name & Value & Units \\
& & \\
\hline Smple_frequency & 5 & $\mathrm{KHz}$ \\
Sampling_points & 1000 & $($ ) \\
Starting_frequency & 200 & $\mathrm{~Hz}$ \\
Modulation_rate & 30 & $($ ) \\
DOA & -12 & $\circ$ \\
\hline
\end{tabular}

(c) Radar_signal_3

\begin{tabular}{ccc} 
Name & Value & Units \\
& & \\
\hline Smple_frequency & 5 & $\mathrm{KHz}$ \\
Sampling_points & 1000 & $($ ) \\
Starting_frequency & 300 & $\mathrm{~Hz}$ \\
Modulation_rate & 40 & $($ ) \\
DOA & 21 & $\circ$ \\
\hline (d) Radar_signal_4 & \\
\hline Name & Value & Units \\
& & \\
\hline Smple_frequency & 5 & $\mathrm{KHz}$ \\
Sampling_points & 1000 & $($ ) \\
Starting_frequency & 400 & $\mathrm{~Hz}$ \\
Modulation_rate & 50 & $($ ) \\
DOA & 50 & $\circ$ \\
\hline
\end{tabular}

Turn LFM signals to the frequency signals. In this experiment, suppose the frequency of oscillator is $2 \mathrm{KHz}$, magnification of amplifier is 2 .

Set the amplifier amplification to establish mixed matrix $A$. In this experiment, $A$ is

$$
A=\left[\begin{array}{cccc}
0.5774 & 0.5774 & 0.5774 & 1.0000 \\
0.2618 & -0.5270 & 0.3260 & -0.9570 \\
-0.3400 & 0.3846 & -0.2092 & 0.8317
\end{array}\right]
$$


So the "Mixed_Signals" can be obtained, and we believe that the mixed signals can be totally intercepted. Put the mixed signals to the simulation system, after treatment of each module, the final results are

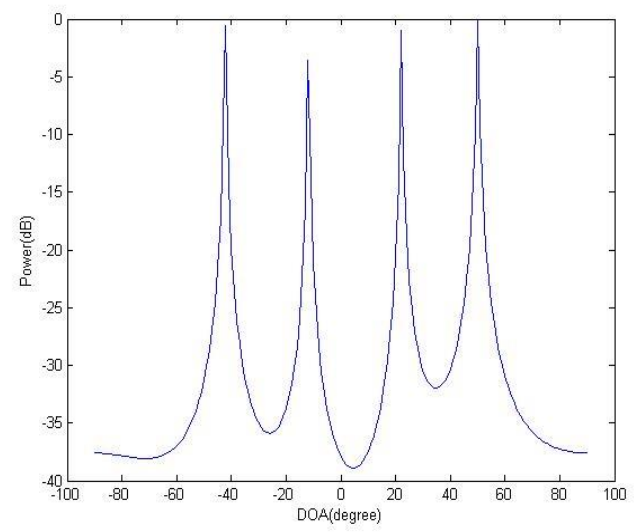

(a) Signal spatial specturm
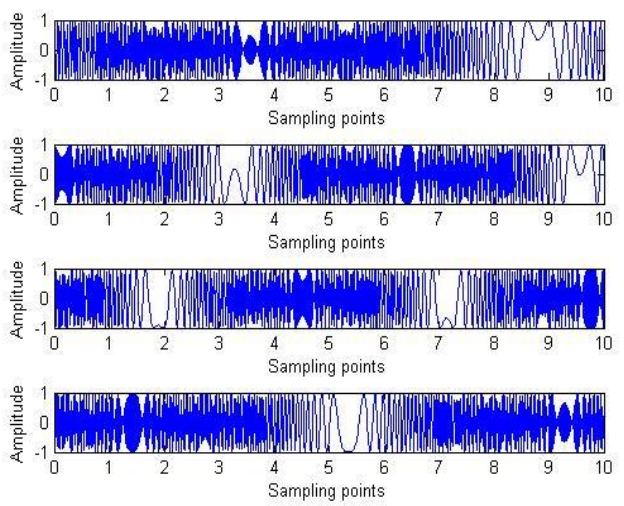

(b) Time-domain waveforms estimation

Figure 8. Results

From the Figure 8(a), we can obtain the number of signals and the estimation of DOA. Traversal search the spatial specturm, figure out the number of peaks and the number of signals is peak numbers. The horizontal coordinates corresponding to the peak value contain the DOA information of each signal, convert them into angle information, so we can get the DOA of each signal, the DOA result from Figure 8(a) is $\left[-41^{\circ}, 49^{\circ},-12^{\circ}, 21^{\circ}\right]$. And Figure 8(b) shows the estimation of time-domain waveforms, and the estimated mixed matrix is

$$
\hat{A}=\left[\begin{array}{cccc}
0.5795 & 0.5784 & 0.5804 & 0.5774 \\
0.2574 & 0.3265 & -0.5513 & -0.5271 \\
-0.3396 & -0.2078 & 0.4782 & 0.3847
\end{array}\right]
$$

Due to the uncertainty of blind signal separation, there are difference between source signals waveforms and estimated waveforms in sequence. But for the radar signal sorting problem, we are more concerned with characteristics than sequence of signals, so the difference can not affect final results. And we define the estimation error to evaluate the estimation accuracy of mixed matrix.

Definition 4. Estimation error

$$
E_{A}=10 \lg \left(\frac{1}{N}\|A-\hat{A}\|_{F}\right)
$$


Where $E_{A}$ is estimation error, \|\|$_{F}$ is F-norm, and the unit of estimation error is dB. So we can see from Eq. (27) that the smaller the $E_{A}$ value, the higher the estimation accuracy.

According to the Definition 4, the diagram of the estimation error with changing signal noise ratio (SNR) is

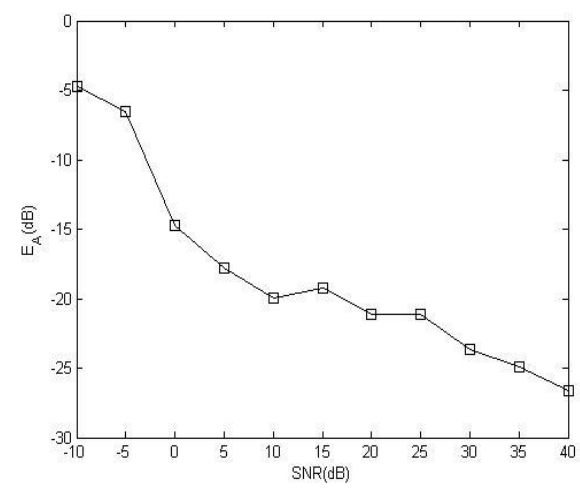

Figure 9. Estimation Error in the Condition of Different SNR

As we can see in Figure 9, the estimation error of mixed matrix is in $-5 \mathrm{~dB}$ below, in the case of large SNR, the error value is very small. So it suggests that the proposed method is effective. And it also shows that the presented simulation system scheme is correct, the system can realize radar signal sorting just like the real one.

\section{Conclusion}

In this paper, a scheme of radar warning receiver modeling system is proposed. The system has three modules, front signal intercept, parameter estimation and signal sorting. And the function, tasks, realizing method of each module is discussed. The innovation of this paper lies in applying the joint parameter estimation method based on cyclic-correlation into parameter estimation module and applying the sorting method based on detection of time-frequency single source point into signal sorting module, both of them can obtain satisfactory results, and there are two advantages of presented methods:

Both of them are in combination with blind signal separation technology, so that they can solve the problem of radar signals sorting under the condition of less priori knowledge.

In a wide range of SNR, the sorting method, presented in the paper, shows good robustness, it indicates that this method can be applied in actual battlefield conditions.

And the results of experiments prove that the simulation system can sort out radar signals from mixed signals in a wide range of SNR. Not only that, the modeling system based on SystemVue can connect the physical equipments to form semi-physical system or regarding this system as a subsystem, apply it to the larger system. So the realization of the radar warning modeling system is of great practical significance, and the system provides a flexible platform for teaching and experiments.

\section{References}

[1] L. David and B. Anurag, "Radar System Design and Interference Analysis Using Agilent SystemVue [Z]", Agilent Technologies Application Note, (2010).

[2] Using Agilent SystemVue to Create Realistic Scenarios for Radar and EW Applications [Z], Agilent Technologies Application Note, (2012). 
[3] Using SystemVue's Radar Library to Generate Signals for Radar Design and Verification [Z], Agilent Technologies Application Note, (2011).

[4] G.-b. Hu and Y. Liu, "Blind Arrival Time Estimation of Signals Based on Correlation and Reversed Accumulation [J]", Journal of Nanjing University of Aeronautics \& Astronautics", vol. 41, no. 3, (2009), pp. 391-396.

[5] J. Kim, H. J. Yang and B. W. Jung, "Blind Calibration for a Linear Array with Gain and Phase Error Using Independent Component Analysis [J]”, IEEE Antennas and Wireless Propagation Letters”, vol. 9, (2010), pp. 1259-1262.

[6] H. Tao and Y.-y. Zhou, "Diffusion features in radar specific emitter identification [J]", Acta Electronica Sinica, vol. 41, no. 3, (2013), pp. 502-507.

[7] H. Sun and H. An, "A Method to Select Radar Signal Based on Blind Source Separation [J]", Modern Radar, vol. 23, no. 3, (2006), pp. 47-50.

[8] C.-y. Qi, Z.-j. Chen and Y.-s. Zhang, "Rapid DOA Estimation Algorithms Based on Projection Pretransformation [J]", Systems Engineering and Electronics, vol. 28, no. 4, (2006), pp. 525-528.

[9] G. Bin, W. L. Woo and S. S. Dlay, "Single-Channel Source Separation Using EMD-Subband Variable Regularized Sparse Features [J]", IEEE Transactions on Audio, Speech, and Language Processing, vol. 19, no. 4, (2011), pp. 961-976.

[10] N. Klaus, O. Esa and O. Hannu, "On the Performance Indices of ICA and Blind Source Separation [C]", 2011 IEEE 12th International Workshop on Signal Processing Advances in Wireless Communications, (2011), pp. 461-465.

[11] Advanced Radar Warning System. www.saabgroup.com.

[12] X.-j. Chen, H. Cheng and B. Tang, "Underdetermined Blind Radar Signal Separation Based on ICA [J]", Journal of Electronics \& Information Technology, vol. 32, no. 4, (2010), pp. 919-924.

[13] B. Lin, Z.-h. Zhang and J.-b. Zhu, "Sparsity Model and Performance Analysis of DOA Estimation with Compressive Sensing [J]", Journal of Electronics \& Information Technology, vol. 36, no. 3, (2014), pp. 589-894.

[14] R J. Wang, Y J. Zhan and H F. Zhou, "Underdetermined Blind Source Separation Based on Null-Space Representation and Maximum Likelihood [J]", J. Commun., vol. 33, (2012), pp. 183-190.

[15] Y. Arie, "Performance Analysis of the Strong Uncorrelating Transformation in Blind Separation of Complex-Valued Sources [J]", IEEE Trans Signal Process., vol. 60, (2012), pp. 478-483.

[16] X.-d. Zhang, "Modern Signal Processing [M]", Beijing: Tsinghua University Press, (2002).

[17] B.-h. Tan and S.-1. Xie, "Underdetermined Blind Separation Based on Source Signals Number Estimation [J]", Journal of Electronics \& Information Technology, vol. 30, no. 4, (2008), pp. 863-867.

[18] L. Wang, Y. Sh and H.-b. Ji, "Specific Radar Emitter Identification Using Multiset Canonical Correlation Analysis [J]", Journal of Xidian University, vol. 40, no. 2, (2013), pp. 164-171.

[19] J. Y. Wu, W. J. Li, J. P. Huang, J. L. Zhang and D. R. Chen, "Key techniques for Mobile Internet: a survey", SCIENTIA SINICA Informationis, vol. 45, no. 1, (2015), pp.45-69.

\section{Author}

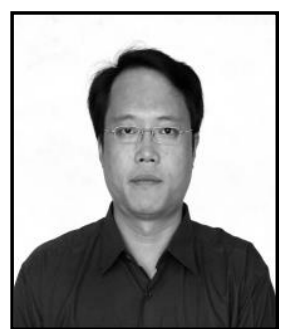

Liu Li-min: (1971-). The main researches are direction for grid computing and computer application. 
International Journal of Control and Automation Vol.8, No.3 (2015) 